\title{
COMMENTARY
}

\section{Weaving a pattern from disparate threads: lamin function in nuclear assembly and DNA replication}

\author{
Christopher J. Hutchison', Joanna M. Bridger', Lynn S. Cox ${ }^{2}$ and lan R. Kill1 \\ ${ }^{1}$ Department of Biological Sciences, The University, Dundee DD1 4HN, UK \\ ${ }^{2}$ CRC Cell Transformation Research Group, Department of Biochemistry, The University, Dundee DD1 4HN, UK
}

\section{SUMMARY}

The major residual structure that remains associated with the nuclear envelope following extraction of isolated nuclei or oocyte germinal vesicles with non-ionic detergents, nucleases and high salt is the lamina (Fawcett, 1966; Aaronson and Blobel, 1975; Dwyer and Blobel, 1976). The nuclear lamina is composed of intermediate filament proteins, termed lamins (Gerace and Blobel, 1980; Shelton et al., 1980), which polymerise to form a basket-weave lattice of fibrils, which covers the entire inner surface of the nuclear envelope and interlinks nuclear pores (Aebi et al., 1986; Stewart and Whytock, 1988; Goldberg and Allen, 1992). At mitosis, the nuclear envelope and the lamina both break down to allow chromosome segregation. As a consequence, each structure has to be rebuilt during anaphase and telophase, allowing cells an opportunity to reposition chromosomes (Heslop-Harrison and Bennett, 1990) and to reorganise looped chromatin domains (Franke, 1974;
Franke et al., 1981; Hochstrasser et al., 1986), which may in turn control the use of subsets of genes. Because of the position that it occupies, its dynamics during mitosis and the fact that it is an essential component of proliferating cells, the lamina has been assigned a number of putative roles both in nuclear metabolism and in nuclear envelope assembly (Burke and Gerace, 1986; Nigg, 1989). However, to date there is little clear cut evidence that satisfactorily explains the function of the lamina in relation to its structure. In this Commentary we will describe some of the recent work that addresses this problem and attempt to provide a unified model for the role of lamins in nuclear envelope assembly and for the lamina in the initiation of DNA replication.

Key words: lamin, nuclear assembly, DNA replication

\section{LAMIN STRUCTURE AND THE REGULATION OF LAMINA ASSEMBLY}

Sequence analysis of cDNA clones encoding lamins has demonstrated that they belong to the intermediate filament supergene family and are now classified as type V intermediate filaments (McKeon et al., 1986; Fisher et al., 1986; Doring and Stick, 1990). As such, all lamins share a common primary structure consisting of a central rod domain comprising three alpha-helical coiled-coil domains, a short amino-terminal globular head domain and a longer, more variable, carboxylterminal globular tail domain (Fig. 1A). The tail domain is important both for transport of the lamins into the nucleus (Loewinger and McKeon, 1988) and for localisation of the lamins at the nuclear envelope (Kitten and Nigg, 1991; Krohne et al., 1989). Higher vertebrates normally express two types of lamin, termed A-type and B-type (Gerace and Blobel, 1980). Of these, B-type lamins are constitutively expressed in all embryonic and somatic tissues (Lehner et al., 1987; Stewart and Burke, 1987; Wolin et al., 1987; Vorburger et al., 1989a; Rober et al., 1989), although different tissues may contain different B-type lamins (Stick and Hausen, 1985; Benevente et al., 1985; Hoger et al., 1988). The expression of A-type lamins is, in contrast, restricted to differentiated tissues (Lehner et al., 1987; Rober et al., 1989). Both A-type and B-type lamins contain a nuclear localisation sequence motif in the tail domain, close to the carboxyl end of the central rod domain (Fig. 1A; Loewinger and McKeon, 1988). Phosphorylation of chicken lamin $B_{2}$ by protein kinase $C$, at a site adjacent to the nuclear localisation sequence, delays translocation of newly synthesised protein across the nuclear envelope (Hennekes et al., 1993). Both types of lamin also contain a sequence motif CaaX (C, cysteine; a, aliphatic amino acid; X, any amino acid) at the carboxyl terminus (Fig. 1A). This sequence serves as a site for modification by isoprenylation (Beck et al., 1988; Wolda and Glomset, 1988; Vorburger et al., 1989b) and methylation (Chelsky et al., 1987). However, A-type lamins and B-type lamins differ in that the modified cysteine residue in the CaaX motif can be removed by proteolytic cleavage of the final 18 amino acids in A-type lamins (Beck et al., 1988; Vorburger et al., 1989b; Weber et al., 1989). In addition, Atype lamins can be synthesised from an alternatively spliced 
mRNA species that lacks codons for the final 82 amino acids (this shortened lamin is termed lamin C; Fisher et al., 1986). Isoprenylation at the CaaX motif is thought to function in positioning the lamins at the nuclear envelope, since the farnesyl residues (the isoprene added to lamins) incorporated at these sites can associate directly with the inner nuclear membrane. Consequently, removal of farnesylated and methylated cysteine residues from A-type lamins, during interphase, may result in these species dissociating from the nuclear envelope at mitosis, whereas B-type lamins, which retain their modified cysteine residues, segregate with nuclear envelope vesicles on nuclear envelope breakdown (Gerace and Blobel, 1980; Stick et al., 1988; Meier and Georgatos, 1994).

Both A-type and B-type lamins contain several putative phosphorylation sites throughout the globular head and tail domains and in the central rod domain. Of these, two sites appear to regulate the state of lamina assembly/disassembly. One site is situated in the amino terminus adjacent to coil 1a, the other in the carboxy terminus adjacent to coil 2 (see Fig. 1A). Lamins will polymerise spontaneously in vitro to form short filaments by head-to-tail assembly (Fig. 1B) and will associate laterally to form paracrystals (Fig. 2B; Aebi et al., 1986; Moir et al.,1991; Heitlinger et al., 1992). Lateral associations, as with cytoplasmic, intermediate filaments, probably occur through antiparallel associations (Quinlan et al., 1989). Lamin paracrystals assembled in vitro can be disassembled by incubation either in cell-free extracts of mitotic cells (Ward and Kirschner, 1990) or with highly purified fractions of $\mathrm{p} 34^{\mathrm{cdc} 2}$ kinase (Peter et al., 1991). The M-phase specific sites of lamin phosphorylation in the amino- and carboxy-terminal domains both conform to the $\mathrm{p} 34^{\mathrm{cdc} 2}$ kinase consensus sequence (Fig. 1A; Peter et al., 1990; Ward and Kirschner, 1990). Furthermore, serine to arginine substitutions at these sites block lamina disassembly at mitosis when cDNAs encoding mutant lamins are transfected into fibroblasts (Heald and McKeon, 1990). As a result of the studies described above, it is widely held that lamina disassembly is controlled directly through phosphorylation by $\mathrm{p} 34^{\mathrm{cdc} 2}$ kinase (Fig. 1B). However, it is still unclear how some associations are made between lamins during lamina reassembly. When a lamina is viewed in situ it is organised as a network (Fig. 2A; Aebi et al., 1986; Stewart and Whytock, 1988), but as yet network assembly of lamins has not been recapitulated in vitro and instead lamins are assembled into paracrystals. Nevertheless, since much is known about the structure of the lamina, can this information be used to determine how the lamina may influence events in nuclear metabolism?

\section{THE ROLE OF LAMINS IN NUCLEAR ENVELOPE REASSEMBLY: LESSONS FROM CELL-FREE EXTRACTS}

\section{The systems}

At mitosis (in all vertebrates and many invertebrates) the nucleus disassembles into its component parts. Thus the nuclear envelope breaks down into Golgi-like vesicles and the nuclear pores are released as precursor subunits. Preceding or concomitant with this event the lamina disassembles, with Atype lamins being released as soluble dimers and tetramers, while B-type lamins generally remain associated with nuclear envelope precursor (NEP) vesicles (Fig. 3). Hence mitotic cells are a cytoplasmic milieu containing all of the components that are required to reassemble a nuclear envelope. Thus it is straightforward to convert a mitotic cell into a cell-free nuclear assembly extract by breaking open the cell, recovering the cytoplasmic fraction and inactivating the cyclin-dependant kinase activities that maintain the mitotic state. To date, such extracts have been derived from synchronously dividing Chinese hamster ovary (CHO) cells (Burke and Gerace, 1986), fertilised and parthenogenically activated amphibian eggs (Lohka and Masui, 1983; Lohka and Maller, 1985; Miake-Lye and Kirschner, 1985) and syncitial Drosophila embryos (Crevell and Cotterill, 1991). Of these, by far the best-characterised extracts are those derived from Xenopus eggs, which are also the most convenient to prepare. Each unfertilised Xenopus egg contains sufficient stores of precursors to assemble up to 12,000 nuclei in the absence of de novo transcription or translation (Forbes et al., 1983) and many thousands of eggs can be collected that are synchronised either in metaphase of second meiosis or in early $G_{1}$ phase of the first

\section{A}
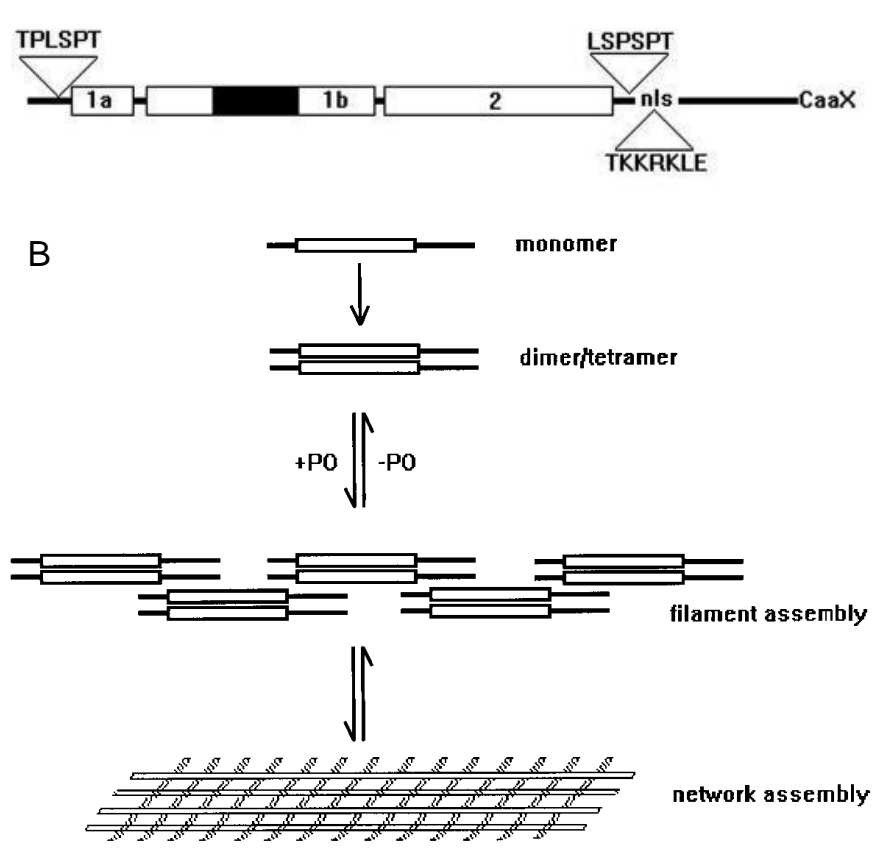

Fig. 1. (A) Structural and functional motifs in a stylised lamin. Boxes labelled $1 \mathrm{a}, 1 \mathrm{~b}$ and 2 represent the alpha-helical coiled-coil domains. The shaded area in coli $1 \mathrm{~b}$ represents additional heptad repeats occuring in lamin polypeptides but not in other intermediate filament proteins. The sequence TPLSPT in the N-terminal globular domain and LSPSPT in the C-terminal globular domain are p34 $4^{\text {cdc2 }}$ kinase target sites. The sequence TKKRKLE is a nuclear localisation signal (nls) sequence. The C-terminal contains a motif, $\mathrm{CaaX}$, which is required for nuclear envelope association. (B) Scheme illustrating the steps in lamina assembly. Lamin monomers form homodimers through associations between the coiled-coil domains. Dimers and tetramers then form filaments by head-to-tail associations and lateral extension. Filament assembly is regulated by

phosphorylation/dephosphorylation with p34 ${ }^{\mathrm{cdc} 2}$ kinase. In vivo, filaments assemble into a network beneath the inner nuclear membrane. 

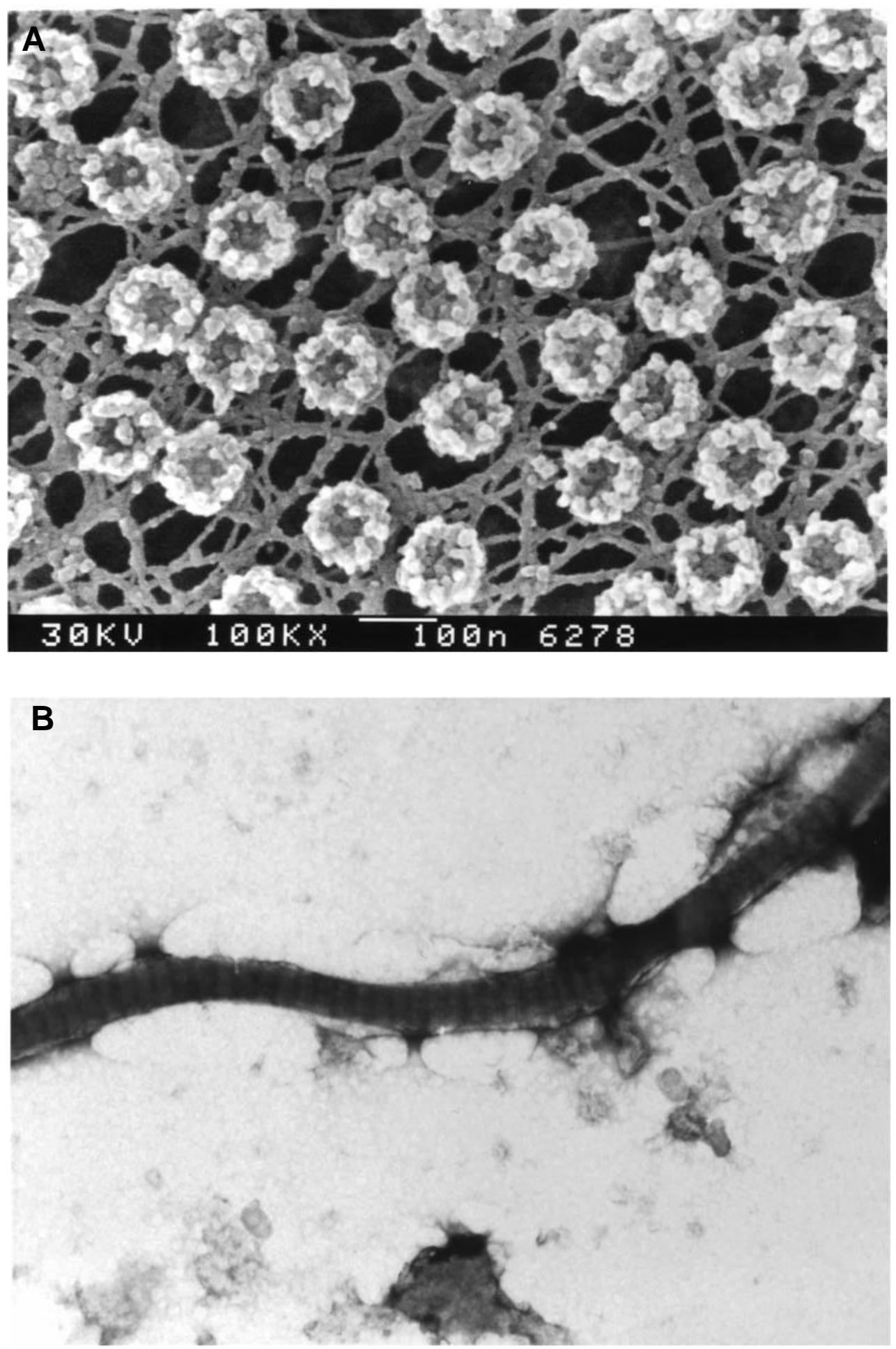

Fig. 2. (A) En face view of a Xenopus oocyte germinal vesicle lamina. Germinal vesicles were manually dissected from stage six oocytes and the nuclear membranes removed by incubation in Triton X-100. The nuclear envelopes were then prepared for high-resolution scanning electron microscopy. In this micrograph, nuclear pores can be seen supported on a basket-weave network of lamin filaments (the micrograph was provided courtesy of Dr Martin Goldberg and Dr Terry Allen, Paterson Institute, Manchester). (B) Lamin paracrystal assembled in vitro. Lamin $\mathrm{B}_{3}$ was purified from Xenopus egg extracts, dialysed from $6 \mathrm{M}$ urea to $250 \mathrm{mM} \mathrm{NaCl}$ and paracrystals were collected on copper grids. The grids were negatively stained with uranyl acetate and viewed with a Joel transmission electron microscope. Lamin paracrystals display a typical repeated pattern of light and dark bands with an axial repeat of $27 \mathrm{~nm}$ (this micrograph was provided courtesy of Hazel Jenkins and Dr Roy Quinlan, University of Dundee). 
mitotic cell-cycle (Lohka and Maller, 1985; Blow and Laskey, 1986). In contrast, while cell-cycle synchrony can easily be induced in $\mathrm{CHO}$ cells, only limited supplies of nuclear envelope precursors are present in each cell (sufficient for the assembly of two nuclei), so that depletion of this pool during preparation of the extract poses a problem. Although cell-free extracts from embryos of genetically tractible organisms such as Drosophila appear attractive, these systems suffer from several limitations. Since each female lays only small eggs, extracts are prepared from several batches of embryos, which are normally collected at the syncitial stage. Because of their very rapid cell cycles the embryos cannot be collected at synchronous stages, and in addition a significant proportion of the maternal pool of nuclear envelope precursors in each embryo will have been depleted. Moreover, from a practical point of view, breaking the chitin shell of the embryos without drastically changing the contents is very tricky. Not surprisingly, nuclear envelope assembly is relatively inefficient in these extracts (Crevel and Cotterill, 1991).

\section{Models for nuclear envelope assembly}

Upon release from a metaphase state, nuclear envelopes reform around either endogenous or exogenous DNA templates in each extract. Three alternative pathways might be used in order to reassemble a functional nuclear envelope. In the first pathway (model 1) soluble lamins first assemble on the surfaces of decondensing chromatin (here the term soluble lamins is preferred to A-type lamins: this is because in early embryos a major fraction of B-type lamins is soluble at mitosis; Firmbach-Kraft and Stick, 1993). This allows NEP vesicles that have associated B-type lamins to bind to the modified chromatin, an event that initiates nuclear envelope vesicle fusion. Nuclear pores are then inserted into the envelope by an unknown mechanism. In the second pathway (model 2), nuclear envelope precursors bind to the surfaces of chromatin independently of soluble lamins. Upon fusion of these vesicles to form double-layered membranes, nuclear pores assemble. Only then do soluble lamins migrate into the nucleus and assemble into a lamina. In the third model (model 3; Fig. 3) no sequential pathway is required. Instead soluble lamins, membrane-associated lamins and NEPs (not associated with lamins) bind cooperatively to the surfaces of chromatin to allow nuclear envelope assembly to occur at an optimal rate. Once the nuclear envelope is formed and nuclear pores are in place, the bulk of soluble lamins can be transported into the nucleus, allowing lamina assembly to occur due to high local concentrations of lamin dimers and tetramers. Can cell-free extracts be used to distinguish between these pathways?

\section{Experimental evidence}

An obvious means of addressing this question is to functionally deplete or remove lamins from cell-free extracts using
Fig. 3. Nuclear envelope assembly and disassembly. Nuclear envelope disassembly occurs at prophase and leads to the dispersal of nuclear membranes as several populations of vesicles, some of which have associated B-type lamins and some of which have no associated lamins. Other lamins are dispersed as dimers and tetramers and are not associated with membrane fractions. Dispersal of nuclear pore subunits is poorly understood. At late anaphase membrane vesicles not associated with lamins, laminassociated membrane vesicles and soluble lamins bind cooperatively to the surface of decondensing chromosomes. As the cell enters telophase, membrane vesicles fuse and nuclear pores are assembled to form a continuous nuclear envelope. Upon completion of nuclear envelope assembly, the bulk of soluble lamins are rapidly imported into the nucleus. Lamina assembly occurs once the lamins have achieved a threshold concentration.

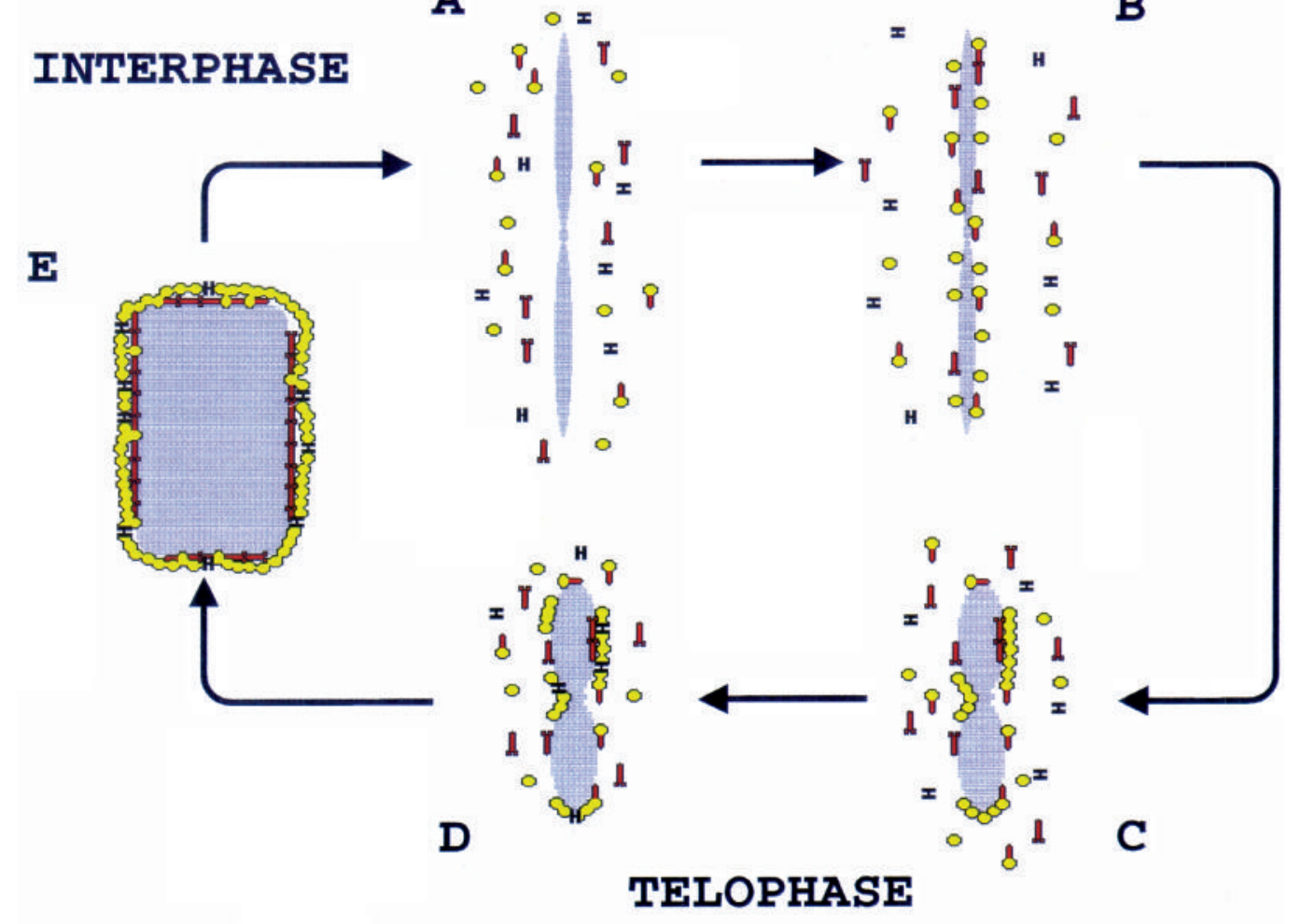

B

A

ANAPHASE

$\begin{aligned} \boldsymbol{T}= & \text { Lamins } \quad=\text { Chromatin } 0=\text { Vesicles } \\ \nabla= & \text { Lamin-associated } \\ & \text { vesicles }\end{aligned}$ 
anti-lamin antibodies. This approach has been tried in five different laboratories with the result that on three occasions lamins were reported to be required for nuclear envelope assembly (Burke and Gerace, 1986; Dabauvalle et al., 1991; Ulitzur et al., 1992) and on two occasions nuclear envelope assembly appeared to occur independently of lamina assembly (Newport et al., 1990; Meier et al., 1991). The first lamin depletion experiments to be performed utilised $\mathrm{CHO}$ cell extracts; A-type lamins or B-type lamins were removed from the extracts using polyclonal antibodies linked to Staphylococcus aureus. In the absence of either A-type lamins or Btype lamins, nuclear envelope assembly was severely limited (Burke and Gerace, 1986). In an independent series of experiments, anti-lamin antibodies were microinjected into $\mathrm{PtK}_{2}$ cells at metaphase. This treatment resulted in cell-cycle arrest at telophase with only partially formed nuclear envelopes (Benevente and Krohne, 1986). These studies led Burke and Gerace (1986) to proposed that nuclear envelope assembly occurred via a two-step mechanism essentially as described in model 1. Further evidence to support this model was derived from experiments in which purified A-type lamins were shown to be capable of assembling efficiently into insoluble structures on the surfaces of isolated metaphase chromosomes (Glass and Gerace, 1990; Burke, 1990).

Cell-free extracts of Drosophila embryos also appeared to require soluble lamins for nuclear envelope assembly. Depletion of the soluble $75 \mathrm{kDa}$ isoform of Drosophila lamin (Smith and Fisher, 1989) from extracts using polyclonal antibodies prevented the attachment of nuclear envelope precursors to the surface of exogenously added sperm chromatin. However, nuclear envelope assembly could be rescued in these extracts by the addition of purified bacterially expressed lamin (Ulitzur et al., 1992).

In contrast to these data, experiments using cell-free extracts of Xenopus eggs have proved equivocal in relation to the role of lamins in nuclear envelope assembly. In an elegant series of experiments, Newport and co-workers (1990) showed that physical depletion of lamin $\mathrm{B}_{3}$ (at that time thought to be the only lamin present in early cleavage embryos) from cytosolic fractions of Xenopus egg extracts did not prevent nuclear envelope assembly, on readdition of untreated membranes, but did result in the formation of small fragile nuclei. In an independent series of experiments, Meier and co-workers (1991) used monoclonal antibodies to functionally deplete lamin $\mathrm{B}_{3}$ from unfractionated Xenopus egg extracts, again with the result that nuclear envelope assembly still occurred. What conclusions can be drawn from these investigations? Newport and coworkers (1990) have proposed a lamin-independent pathway for nuclear envelope assembly that is similar to model 2 . In support of this model, several groups have demonstrated that not only can NEPs bind to chromatin in the absence of lamins but they can also fuse (Pfaller et al., 1991; Vigers and Lohka, 1991; Newport and Dunphy, 1992; Boman et al., 1992). However, interpretation of these experiments is potentially misleading, as is interpretation of experiments that show that A-type lamins coat the surfaces of chromosomes (Glass and Gerace, 1990; Burke, 1990), for the following reasons. Regardless of the order in which lamins and membrane vesicles associate with chromatin, it is clear that both can and do so. Thus in minimal systems in which chromosomes are mixed with lamins, or chromatin is mixed with nuclear envelope pre- cursors, associations will occur. Therefore, minimal systems cannot be used to define the order of steps in the pathway to nuclear envelope assembly without reference to more complex systems. The problem of interpreting results from cell-free systems is highlighted by results described by Dabauvalle and co-workers (1991). In contrast to earlier reports, this group demonstrated that functional depletion of lamin $\mathrm{B}_{3}$ in Xenopus egg extracts did prevent nuclear envelope assembly. However, a simple hypothesis can accommodate the apparent contradiction between the results of Meier, Newport and Dabauvalle. Lamin $\mathrm{B}_{3}$ has been reported as the only lamin present in early cleavage embryos (Stick and Hausen, 1985) and whilst lamin $\mathrm{B}_{3}$ is undoubtedly B-type (Stick, 1988) it does not remain associated with membrane vesicles at mitosis (Firmbach-Kraft and Stick, 1993). If a small, previously undetected fraction of membrane-associated lamin is also present in Xenopus egg extracts, the experiment performed by Newport and coworkers must be re-interpreted, since only soluble lamins were depleted from their extracts. Furthemore, it is possible to explain the apparent discrepancy between the results of Meier et al. (1991) and Dabauvalle et al. (1991) if the antibody used by Meier prevents assembly of soluble lamins into a lamina while the antibody used by Dabauvalle prevents the binding of membrane-associated lamins to chromatin.

\section{RESOLVING THE DISCREPANCIES}

To address the points raised above, two independent studies have recently been reported. In the first of these, Jenkins and co-workers (1993) developed a novel approach to remove lamins physically from unfractionated egg extracts using antibodies linked to magnetic beads. The monoclonal antibodies used in this study recognised all three B-type lamins expressed during Xenopus embryogenesis (Stick and Hausen, 1985; Doring and Stick, 1990). The results of the experiments indicate that, after removing $>96 \%$ of lamins from Xenopus egg extracts, nuclear envelope assembly still occurred. However, in a detailed series of analyses, Lourim and Krohne (1993) used a panel of monoclonal antibodies to re-investigate the lamin composition of Xenopus egg extracts. They identified a hitherto undetected membrane-associated fraction of $\mathrm{B}_{2-}$ like lamin. In addition, they also detected a minor fraction of lamin $\mathrm{B}_{3}$ associated with membrane vesicles. The monoclonal antibodies used by Jenkins et al. (1993) had previously failed to detect any lamins in partially purified fractions of NEPs (Vigers and Lohka, 1992) and consistently failed to detect lamin $\mathrm{B}_{2}$ in egg extracts. Thus while the result described by Jenkins et al. (1993) indicates that soluble lamins are not essential for the initial events of nuclear envelope reassembly, Lourim and Krohne (1993) suggest that previously undetected insoluble (membrane-associated) lamin fractions are present in egg extracts and it is these lamins that are essential for NE assembly. Significantly, despite the failure to detect lamins in depleted extracts using immunoblotting and immunoprecipitation, nuclei assembled in the same extract do contain a residual lamina (5\% of the normal lamin complement; Jenkins et al., 1993). The observations of Lourim and Krohne (1993) are the first indication of the complexity of membrane fractions defined as NEPs. It therefore seems likely that NEPs represent an heterogeneous population. In a recent series of experiments, 
Foisner and Gerace (1993) described the behaviour of four integral nuclear membrane proteins during mitosis. Each protein had been shown to line the nucleoplasmic face of the inner nuclear membrane (Senior and Gerace, 1988; Foisner and Gerace, 1993). In addition, these proteins were shown to have lamin-binding activity and were therefore termed lamina-associated proteins, or LAPs. Indirect immunofluorescence revealed that two of the LAPs re-associated with chromosomes at anaphase, whilst B-type lamins reassociated with chromosomes at telophase. Complementary results have also been reported by Chaudhary and Courvalin (1993). These highly suggestive results allow us to accommodate all of the available information into a unified model. If it is assumed that inner NEPs are of two types, those associated with B-type lamins and those associated with LAPS, it can be inferred that each can bind to the surface of decondensing chromatin in the absence of soluble lamins. However, in vivo each membrane fraction probably binds cooperatively with soluble lamins to optimise the rate of nuclear envelope assembly. Nevertheless, the removal of soluble lamins from cell-free systems that have an over-abundance of NEP vesicles might not affect the final outcome of experiments designed to test whether nuclear envelope assembly could occur or not. Here of course is the crux of the argument. Only in the Xenopus system can an overabundance of NEPs be ensured. Moreover, since all cell-free extracts deteriorate during incubation, there is only a limited window of opportunity during which nuclear envelope assembly can occur. This window of opportunity has only rarely been documented (Hutchison et al., 1989; Blow, 1993) but its implications are far-reaching. If, as we propose, the model shown in Fig. 3 is correct, then depleting soluble lamins from all but the Xenopus egg extract is likely to limit severely the rate of nuclear envelope assembly. However, by prolonging nuclear envelope assembly in both the $\mathrm{CHO}$ cell-free system and the Drosophila cell-free system, the window of opportunity for complete nuclear envelope assembly is lost.
Thus we propose that soluble lamins do contribute to nuclear envelope assembly in vivo and that discrepancies between results in cell-free extracts are a reflection of the relative sizes of nuclear envelope precursor pools and also of the stability of each extract. How then should investigations with cell-free extracts be conducted in the future? Currently, we and others are characterising the protein composition of membrane fractions that bind to the surfaces of chromatin. These studies should lead to the identification and molecular analyses of families of chromatin receptors. Determination of the kinetics of the associations made between each receptor and chromatin, or between different receptors (including lamins) in biochemically defined systems, will clarify the order in which different membrane fractions become associated with chromatin. Additionally, genetically tractible systems such as Zebra fish could usefully be exploited to provide similar evidence in living cells.

\section{THE ROLE OF LAMIN A IN NUCLEAR ENVELOPE ASSEMBLY}

In the preceding discussion we proposed that the most likely pathway for the initial events of nuclear envelope assembly involve co-operative interactions between different NEP fractions and the surfaces of decondensing chromatin. Furthermore, whilst involving lamins these interactions occur prior to the formation of an extensive lamina. This model is supported by several lines of investigation. Indirect immunofluorescence microscopy indicates that in Xenopus cell-free extracts (Hutchison et al., 1988), CHO cells (Foisner and Gerace, 1993) and human dermal fibroblasts (HDF; Bridger et al., 1993) an extensive lamina is not formed until late in telophase or early in $\mathrm{G}_{1}$ phase of the cell-cycle. This is particularly apparent when observing the behaviour of A-type lamins. In a carefully performed series of experiments,

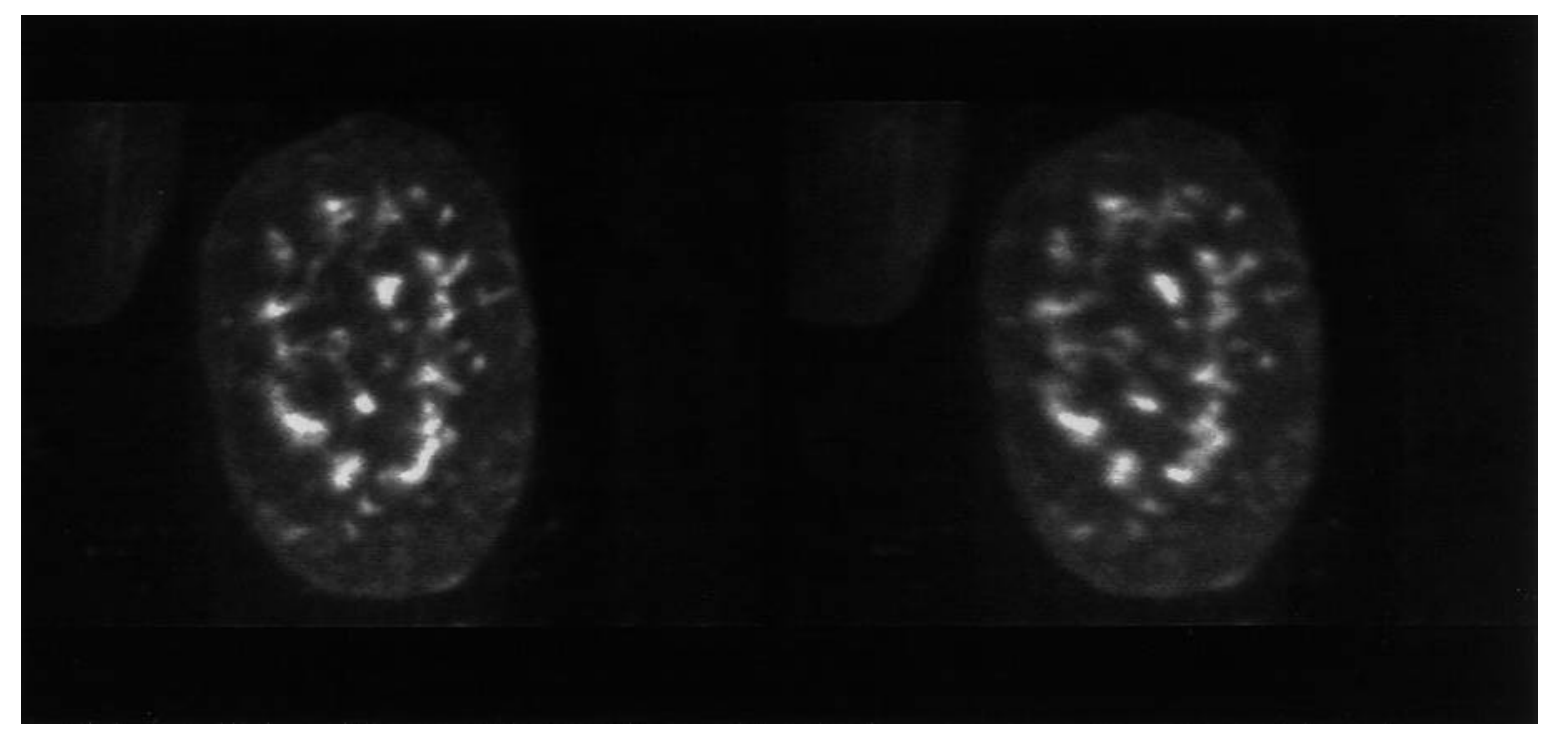

Fig. 4. Stereo pairs showing internal lamin foci in $\mathrm{G}_{1}$-phase human dermal fibroblasts. Indirect immunofluorescence was performed on $\mathrm{G}_{1}$ phase human dermal fibroblasts using anti-lamin A antibodies. Z-series were collected at $0.5 \mu \mathrm{m}$ intervals through a representative cell and projected as stereo pairs. A three-dimensional effect can be achieved by fusing each image in the centre of the micrograph with relaxed viewing. 
Bridger and co-workers (1993) used confocal microscopy to demonstrate that A-type lamins first appeared in the nuclei of $\mathrm{G}_{1}$ fibroblasts as a series of spots and fibrils that accumulated at sites deep within the nucleoplasm (Fig. 4). The majority of these fibrils do not associate with nuclear membranes but do associate with partially decondensed chromatin. Late in $G_{1}$ internal lamin staining disappears, giving way to a perinuclear pattern. Subsequently, the distribution of A-type lamins and B-type lamins was compared in the same cells. While A-type lamins were predominantly located in the nucleoplasm, Btype lamins were concentrated at the nuclear periphery ( $\mathrm{J}$. Bridger, unpublished data). Similar structures to those reported by Bridger et al. (1993) were also observed when biotinylated bacterially expressed human lamin A was microinjected into the cytoplasm of Swiss 3T3 fibroblasts (Goldman et al., 1992). Apparently, microinjected lamins are translocated rapidly across the nuclear envelope but are only slowly incorporated into a peripheral lamina. As a result, intermediate structures accumulate in the nucleoplasm. The results described by Goldman and co-workers (1992) thus provide a clear explanation for the observations of Bridger et al. (1993). Therefore, if nuclear envelope assembly is completed prior to the accumulation of A-type lamins, the large cytoplasmic pool of these lamins present in $G_{1}$ cells would be rapidly transported across the nuclear envelope. Since the transport of A-type lamins into the nucleus appears to be considerably faster than their rate of incorporation into the lamina, they would initially polymerise at nucleoplasmic sites (Bridger et al., 1993).

\section{THE PARADOX OF ISOPRENYLATION}

Despite this evidence, our model does not fully accommodate studies concerning the role of isoprenylation in lamina assembly. As discussed above, lamina assembly appears to require specific modifications at the CaaX motif (Beck et al., 1988; Wolda and Glomset, 1988; Pollard et al., 1990). Chicken lamin B2 (Vorburger et al., 1989b), human lamin B (Farnsworth et al., 1989), Xenopus lamin B 3 (Firmbach-Kraft and Stick, 1993) and human lamin A (Sinensky et al., 1994) are all modified by farnesylation and methylation on the cysteine residue of the CaaX motif. Following modification of B-type lamins, the three carboxy-terminal amino acids are removed by proteolysis leaving a hydrophobic carboxy terminus. Lamin A is also modified by proteolysis, which in this instance appears to be directed by a nuclear protease that removes the final 18 amino acids including the modified cysteine residue (Weber et al., 1989; Beck et al., 1990).

CaaX modifications have been shown to be important for correct assembly of the lamina, by examination of certain mutant forms. Cysteine to alanine substitutions in the CaaX motif of chicken lamin $B_{2}$ prevents farnesylation and methylation. When expressed in mouse L cells, the mutant chicken lamin is still imported into nuclei, but its association with the nuclear envelope is delayed by 24 hours when compared with wild-type lamins (Kitten and Nigg, 1991). Site-specific mutagenesis of human lamin B also suggests that the CaaX motif acts in conjunction with the nuclear localisation signal sequence to direct this lamin to the nucleus (Holtz et al., 1989). Following mitosis, the nuclear envelope and lamina must reassemble, using a pre-existing pool of already posttranslationally modified lamin A. This raises the question of how much mature lamin A can be relocated to the nuclear envelope during telophase. To address this question Krohne and co-workers (1989) injected mammalian lamins into the cytoplasm of Xenopus oocytes and monitored their subsequent redistribution to the nucleus. They reported that, whereas microinjected lamin $\mathrm{C}$ remains in the cytoplasm, a chimaeric lamin consisting of the lamin A CaaX sequence spliced onto the carboxy terminus of lamin C could translocate to the nucleus. In order to accommodate all of these results, it must be argued that at telophase either the bulk of A-type lamins associate with chromosomes prior to nuclear envelope assembly, or that some A-type lamins (those inherited from the preceding interphase) are transported into the nucleus and located at the nuclear periphery of $\mathrm{G}_{1}$ cells by virtue of their association with other proteins. Indirect immunofluorescence clearly indicates that the bulk of lamins do not reassemble on telophase chromosomes. Further experimental support for this view is again derived from cell-free extracts of Xenopus eggs. Using antibodies against nucleoporins, Dabauvalle and co-workers (1990) were able to prevent nuclear pore assembly but not nuclear envelope assembly in Xenopus egg extracts. The resulting nuclei lacked detectable lamins, indicating that the lamina is formed as a consequence of transport through functional nuclear pores. Thus we must assume that most A-type lamins inherited after mitosis (i.e those lacking a CaaX motif) are chaperoned to the nuclear envelope during $\mathrm{G}_{1}$. Furthermore, chaperonins may be of two types, those that aid the transport of lamins into the nucleus and those that subsequently locate the lamins at the nuclear periphery (Fig. 5). This hypothesis could explain the presence of internal lamin foci, which we have observed in $\mathrm{G}_{1}$ cells. Kitten and Nigg (1991) have estimated that lamin $\mathrm{B}_{2}$ is processed and located at the nuclear envelope within 30 minutes of synthesis. In contrast, Bridger et al. (1993) could detect internal nucleoplasmic Atype lamins for up to 10 hours after the end of mitosis. The delay in nuclear envelope association may reflect the increased time required for chaperonins to relocate the relatively large A-type lamin structures that accumulate in $\mathrm{G}_{1}$ nuclei. The restriction of these structures to $G_{1}$ nuclei suggests that lamins synthesised and transported into the nucleus during S-phase are initially unprocessed and therefore can rapidly accumulate at the nuclear periphery.

\section{THE ROLE OF LAMINS IN DNA REPLICATION}

We and others have manipulated Xenopus egg extracts in order to construct nuclei that lack a significant lamina. This has allowed us to examine the role of lamins in nuclear metabolism. Nuclei assembled in Xenopus egg extracts act as independent units of DNA replication (Blow and Watson, 1987) within which semi-conservative DNA replication is initiated and completed on both chromosomal DNA and plasmid DNA (Blow and Laskey, 1986). An essential requirement for the initiation of DNA replication in these extracts is the completion of nuclear envelope assembly (Sheehan et al., 1988). Nuclear envelopes not only help to coordinate initiation events within nuclei (Leno and Laskey, 1991) but also regulate the 


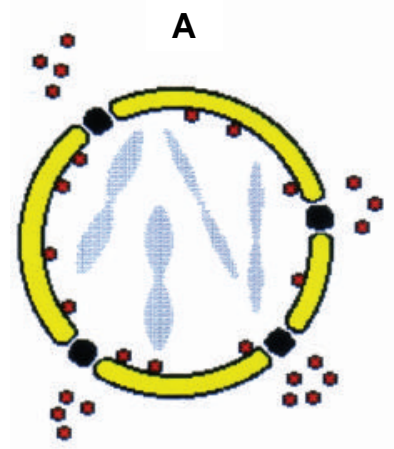

B

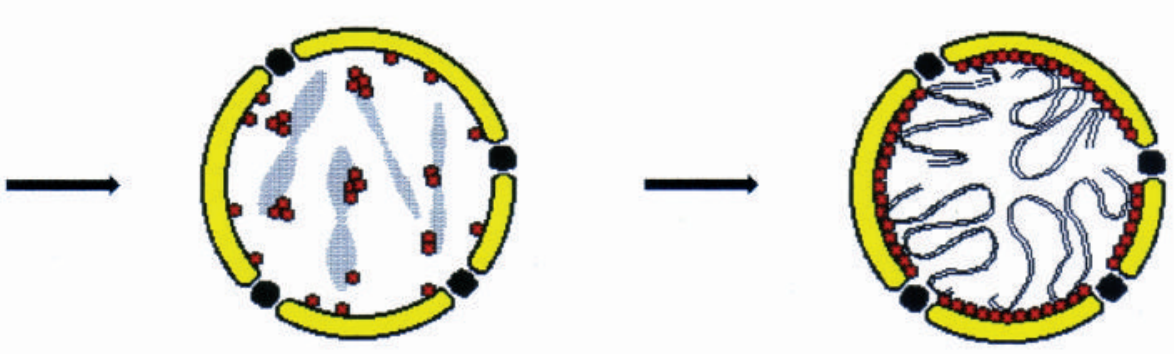

C

Early $\mathbf{G}_{1}$

Late $\mathbf{G}_{1}$

$\square=$ membranes $\quad$ 耕 = chromatin $\quad \square=$ nuclear pores

Fig. 5. Putative pathway for the incorporation of A-type lamins into the nuclear envelope of $\mathrm{G}_{1}$ cells. Upon completion of nuclear envelope assembly at telophase, soluble A-type lamins are rapidly transported into the nucleus. Thus in early $\mathrm{G}_{1}$-phase nuclei the rate at which lamins are accumulated in the nucleoplasm exceeds the rate at which lamins can be incorporated into the nuclear envelope. Initially these lamins polymerise on partially decondensed chromosomes to form nucleoplasmic foci. With time many of the nucleoplasmic lamins become relocated to the nuclear periphery where they contribute to the establishment of looped chromatin domains.

cell-cycle control of DNA replication (Blow and Laskey, 1988; Leno et al., 1992). It now seems likely that cell-cycle regulation of DNA replication is achieved because an activity termed 'licensing factor' cannot cross the NE during interphase (Blow and Laskey, 1988). It is not obvious how initiation events are synchronised by nuclear envelope assembly, but pronuclear formation in lamin-depleted extracts may provide the key. Nuclei that lack a lamina are unable to initiate semiconservative DNA replication (Newport et al., 1990; Meier et al., 1991). For DNA replication to be initiated and completed, nuclear transport must occur efficiently throughout S-phase (Cox, 1992). Therefore, one trivial explanation for the failure of lamina-deficient nuclei to replicate DNA is that they are unable to accumulate karyophilic proteins. With this in mind, Jenkins et al. (1993) compared nuclear transport in normal nuclei and in nuclei that lack a lamina. Surprisingly few differences were detected in either the rate of nuclear transport or the range of karyophilic proteins accumulated. Even more surprising was the observation that the major protein species associated with nuclei that lack a lamina were insoluble following extraction
A

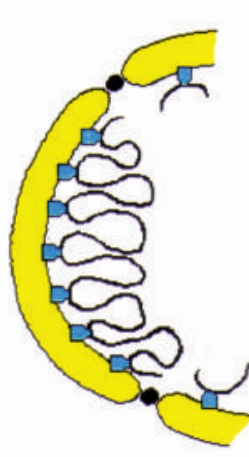

B

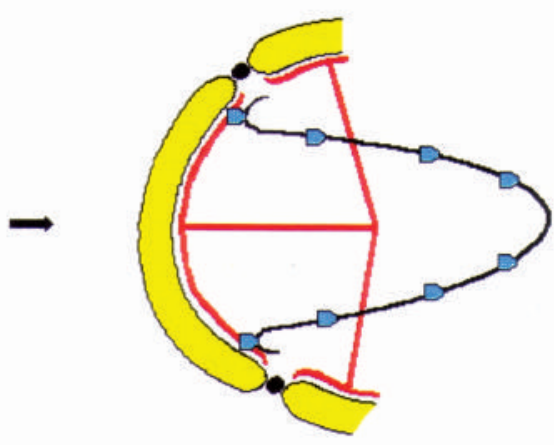

C

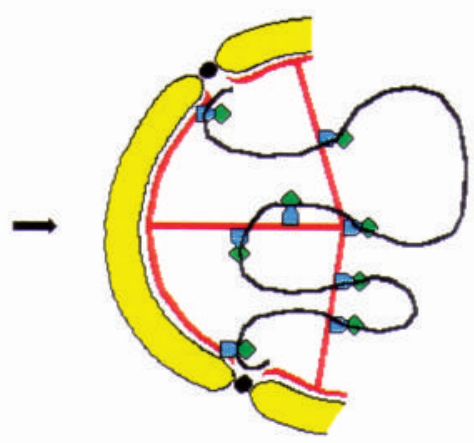

$$
\begin{array}{|lll|}
\hline-=\text { Nuclear matrix } & \bullet=\text { Nuclear pores } & D=\text { SAR } \\
-=\text { Chromatin } & \square=\text { Membranes } & \bullet=\text { PCNA } \\
\hline
\end{array}
$$

Fig. 6. The involvement of lamins in DNA replication. (A) During the initial stages of nuclear envelope assembly, nuclear membranes interact with the entire surface of dencondensing chromosomes, restricting chromatin attachment sites to the nuclear envelope (Newport and Dunphy, 1992). (B) Upon lamin import and assembly, many attachment points between chromatin and the inner nuclear membrane are dislodged. (C) Lamina assembly or lamins is/are required for the formation of a nucleoskeleton. Once the nucleoskeleton is formed looped chromatin domains are re-established by interaction between sequences resembling SARs and the nucleoskeleton. These sites act as replication centres and recruit proteins such as PCNA. 
with detergents and nucleases. Thus it seems unlikely that DNA replication is prevented in lamina-deficient nuclei because nuclear transport is impaired.

One characteristic of DNA replication in Xenopus egg extracts is the clustering of replication forks into multiple foci or centres of DNA replication (Mills et al., 1989; Cox and Laskey, 1991), which model the foci observed in intact and permeabilized mammalian cells (Nakamura et al., 1986; Nakayasu and Berezney, 1989). Preceding the formation of replication centres, replication proteins such as the proliferating cell nuclear antigen (PCNA) and RP-A assemble at prereplication foci, which resemble the subsequent sites of DNA replication (Kill et al., 1991; Adachi and Laemmli, 1992). Although nuclei that lack a lamina import PCNA, the PCNA is not assembled at pre-replication foci and remains soluble (Meier et al., 1991; Jenkins et al., 1993). On the basis of this observation, Jenkins et al. (1993) have proposed that the lamina coordinates initiation events in the nucleus by influencing the formation of replication origins. How could a structure such as the lamina regulate the assembly of such origins? In nuclei assembled in vitro, approximately 100,000 active replication forks are required for DNA replication to be completed in one hour (Blow and Watson, 1987). Since initiation events occur synchronously in this experimental system (Blow and Watson, 1987; Mills et al., 1989; Kill et al., 1991), biotinylated analogues of thymidine can be used to determine the initial position of replication centres in nuclei assembled in vitro. Using confocal microscopy Mills et al. (1989) demonstrated that most replication centres occur at sites that are distant from the nuclear envelope. Thus it must be assumed that the majority of replicons are not directly associated with the lamina. Indeed, the distance of the furthest replication centre from the lamina at initiation is $\sim 5 \mu \mathrm{m}$ (Hutchison, 1994). Furthermore, resinless section EM has revealed that replication centres or factories are attached to filaments of the nucleoskeleton and not to a peripheral lamina (Hozak et al., 1993). Therefore, the only plausible explanation of the result of Jenkins et al. (1993) is that disruption of the lamina or removal of lamins prevents correct assembly of the nucleoskeleton. Since we have now established that immunopurified lamin $\mathrm{B}_{3}$ will rescue DNA replication in lamin-depleted egg extracts (Jenkins et al., 1994), we are confident that absence of lamins directly affects the assembly of a nucleoskeleton. Furthermore, there is a striking temporal correlation between lamina assembly and the initiation of DNA replication in nuclei assembled in vitro (Hutchison et al., 1988).

Could lamins be components of the nucleoskeleton and therefore provide sites for replication origin assembly? Four important observations support this hypothesis. (1) Chromatin domains form position-independent transcriptional units that are physically linked to structures within the nucleus through sequences termed Scaffold Attachment Regions (reviewed by Gasser, 1991). It is now established that SARs will bind directly to B-type lamin dimers, tetramers and polymers in vitro (Luderus et al., 1992). (2) Some SARs act as Autonomously Replicating Sequences in budding yeast (Amati and Gasser, 1990). (3) The clearest resinless section EM images of nucleoskeletons indicate that replication foci (or factories) are supported between fibres that are morphologically identical to intermediate type filaments (Jackson and Cook, 1988; Hozak et al., 1993). (4) The only intermediate filament proteins detected in the nucleus by immunoblotting are lamins (Belgrader et al., 1991).

Two recent reports indicate that lamins do accumulate as filaments in the nucleoplasm (Goldman et al., 1992; Bridger et al., 1993). However, nucleoplasmic lamin filaments are only detectable during $G_{1}$ phase of the cell cycle. Nevertheless, at least some A-type lamins lack the necessary amino acid sequences for nuclear envelope association (see above). Furthermore, in primary cultures of human dermal fibroblasts a fraction of A-type lamins displays different solubility properties when compared to B-type lamins (I.R. Kill and C.J. Hutchison, unpublished data). Is this A-type lamin fraction inherited from the previous interphase and is it located in the nucleoplasm? Perhaps the only way to resolve this question is to incorporate into the nucleus a lamin whose sub-nuclear distribution can be detected without recourse to antibody staining. Micro-injection of biotinylated lamins into proliferating fibroblasts appears to be one way forward (Goldman et al., 1992). Alternatively, microinjection of human lamin A labelled with a fluorescent cyanine dye into human dermal fibroblasts allows direct visualisation of the distribution of Atype lamins (J. Bridger, unpublished data). This approach should now allow us to compare the relative distributions of lamins with or without a CaaX motif in unfixed living cells. We predict that lamins lacking a CaaX motif will form part of the nucleoskeleton. In the light of this prediction we propose that synchronous initiations occur in nuclei assembled in vitro as follows: upon nuclear envelope assembly, chromatin attachment sites are initially tightly associated with the inner nuclear membrane (Fig. 6A: Newport and Dunphy, 1992). Lamin import dislodges many chromatin attachment sites from the inner nuclear membrane as the nuclear lamina and nucleoskeleton are formed (Fig. 6B). Looped chromatin domains are then established by reassociation of sequences resembling SARs to the nucleoskeleton. These sequences act as replication origins and replication proteins such as PCNA rapidly accumulate at sites of SAR-nucleoskeleton interaction, allowing replication to proceed (Fig. 6C).

\section{CONCLUSIONS}

So what do lamins do? Firstly, they provide a passive structural framework underlying and supporting the nuclear envelope and contributing to nuclear envelope assembly. Additionally, we propose that lamins are integral components of the nucleoskeleton, providing a scaffold for precise organisation of chromatin into functional domains, and thereby regulating DNA replication from fixed sites or factories. Thus lamins are essential structural proteins of the nucleus that play an important part in regulating nuclear metabolism.

We thank Dr Terry Allen and Dr Martin Goldberg for supplying scanning electron micrographs; and Miss Hazel Jenkins and Dr Roy Quinlam for supplying us with micrographs of paracrystals. We are also grateful to the Cancer Research Campaign, the Royal Society of Edinburgh and the Wellcome Trust for financial support. 


\section{REFERENCES}

Aaronson, R. P and Blobel, G. (1975). Isolation of pore complexes in association with a lamin. Proc. Nat. Acad. Sci. USA 72, 1007-1011

Adachi, Y. and Laemmli, U. K. (1992). Identification of nuclear prereplication centres poised for DNA synthesis in Xenopus egg extracts: immunolocalisation study of replication protein A. J. Cell Biol. 119, 1-15.

Aebi, U., Cohn, J., Buhle, L. and Gerace, L. (1986). The nuclear lamina is a meshwork of intermediate-type filaments. Nature 323, 560-564.

Amati, B. and Gasser, S. M. (1990). Drosophila Scaffold Attachment Regions bind nuclear matrix and can function as ARS elements in both budding and fission yeast. Mol. Cell Biol. 10, 5442-5454.

Beck, L. A., Hosick, T. J. and Sinensky, M. (1988). Incorporation of a product of mevalonic acid metabolism into proteins of chinese hamster ovary cell nuclei. J. Cell Biol. 107, 1307-1316.

Beck, L. A., Hosick, T. J. and Sinesky, (1990). Isoprenylation is required for the processing of the lamin A precursor. J. Cell Biol. 110, 1489-1499.

Belgrader, P., Siegel, A. J. and Berezney, R. (1991). A comprehensive study on the isolation and characterization of the HeLa S3 nuclear matrix. J. Cell Sci. 98, 281-291

Benevente, R., Krohne, G. and Franke, W. W. (1985). Cell type-specific expression of nuclear lamina proteins during development of Xenopus laevis. Cell 41, 177-190.

Benevente, R. and Krohne, G. (1986). Involvement of nuclear lamina in postmitotic reorganisation of chromatin as demonstrated by microinjection of lamin antibodies. J. Cell Biol. 103, 1847-1854.

Blow J. J. and Laskey, R. A. (1986). Initiation of DNA replication in nuclei and purified DNA by a cell-free extract of Xenopus eggs. Cell 47, 577-587.

Blow, J. J. and Watson, J. V. (1987). Nuclei act as independent and integrated units of replication in a Xenopus cell-free DNA replication system. EMBO J. 6, 1997-2002

Blow, J. J. and Laskey, R. A. (1988). A role for the nuclear envelope in controlling DNA replication within the cell cycle. Nature 332, 546-548.

Blow, J. J. (1993). Preventing re-replication of DNA in a single cell cycle: evidence for a replication licensing factor. J. Cell Biol.122, 993-1002

Boman, A. L., Delannoy, M. R. and Wilson, K. L. (1992). GTP hydrolysis is required for vesicle fusion during nuclear envelope assembly in vitro J. Cell Biol. 116, 281-294.

Bridger, J. M., Kill, I. R., O'Farrell, M. and Hutchison, C. J. (1993) Internal lamin structures within $\mathrm{G}_{1}$ nuclei of human dermal fibroblasts. $J$. Cell Sci. 104, 297-306

Burke, B. and Gerace, L. (1986). A cell-free system to study reassembly of the nuclear envelope at the end of mitosis. Cell 44, 639-652.

Burke, B. (1990). On the cell-free association of lamins A and C with metaphase chromosomes. Exp. Cell Res. 186, 169-176.

Chaudhary, N. and Courvalin, J.-C. (1993). Stepwise reassembly of the nuclear envelope at the end of mitosis. J. Cell Biol. 122, 295-306.

Chelsky, D., Olson, J. F. and Koshland, D. E. (1987). Cell cycle-dependent methyl esterification of lamin B. J. Biol. Chem. 262, 4303-4309.

Cox, L. S. and Laskey, R. A. (1991). DNA replication occurs at discrete sites in pseudonuclei assembled from purified DNA in vitro. Cell 66, 271-275.

Cox, L. S. (1992). DNA replication in cell-free extracts from Xenopus eggs is prevented by disrupting nuclear envelope function. J. Cell Sci. 101, 43-53.

Crevell, G. and Cotterill, S. (1991). DNA replication in cell-free extracts from Drosophila melanogaster. EMBO J. 16, 4361-4369.

Dabauvalle, M.-C., Loos, K. and Scheer, U. (1990). Identification of a soluble precursor complex essential for nuclear pore assembly in vitro. Chromosoma 100, 56-66.

Dabauvalle, M.-C., Loos, K., Merkert, H. and Scheer, U. (1991). Spontaneous assembly of pore complex-containing membranes (annulate lamellae) in Xenopus egg extract in the absence of chromatin. J. Cell Biol. 112, 1073-1082.

Doring, V. and Stick, R. (1990). Gene structure for nuclear lamin Liii of Xenopus laevis: a model for the evolution of IF proteins from a lamin-like ancestor. EMBO J. 9, 4073-4081.

Dwyer, N. and Blobel, G. (1976). A modified procedure for the isolation of a pore compex-lamina fraction from rat liver nuclei. J. Cell Biol. 70, 581-591.

Farnsworth, C. C., Wolda, S. L., Gelb, M. H. and Glomset, J. A. (1989): Human lamin B contains a farneslyated cysteine residue. J. Biol. Chem. 264, 20422-20429.

Fawcett, D. W. (1966). On the occurrence of fibrous lamina on the inner aspect of the nuclear envelope in certain cells of vertebrates. Am. J. Anat. 119, 129146.

Fisher, D. Z., Chaudhary, N. and Blobel, G. (1986). cDNA sequencing of nuclear lamins A and $\mathrm{C}$ reveals primary and secondary structural homology to intermediate filaments. Proc. Nat. Acad. Sci. USA 83, 6450-6454.

Firmbach-Kraft, I. and Stick, R. (1993). The role of CaaX-dependent modifications in membrane association of Xenopus nuclear lamin $\mathrm{B}_{3}$ during meiosis and the fate of B3 in transfected mitotic cells. J. Cell Biol. 123, 16611670.

Foisner, R. and Gerace, L. (1993). Integral membrane proteins of the inner nuclear envelope interact with lamins and chromosomes, and binding is modulated by mitotic phosphorylation. Cell 73, 1267-1279.

Forbes, D. J., Kirschner, M. W. and Newport, J. W. (1983). Spontaneous formation of nucleus-like structures around bacteriophage DNA microinjected into Xenopus eggs. Cell 34, 13-23.

Franke, W. W. (1974). Structure, biochemistry and functions of the nuclear envelope. Int. Rev. Cytol. 4 (suppl) 71-236.

Franke W. W., Scheer, U., Krohne, G. and Jarasch, E. (1981). The nuclear envelope and the architecture of the nuclear periphery. J. Cell Biol. 91, 39s$50 \mathrm{~s}$

Gasser, S. M. (1991). Replication origins, factors and attachment sites. Curr. Opin. Cell Biol. 3, 407-413.

Gerace, L. and Blobel, G. (1980). The nuclear envelope lamina is reversibly depolymerised during mitosis. Cell 19, 277-287.

Glass, J. R. and Gerace. L. (1990). Lamins A and C bind and assemble at the surface of mitotic chromosomes. J. Cell Biol. 111, 1047-1057.

Goldman, A. E., Moir, R. D., Montag-Lowry, M., Stewart, M. and Goldman, R. D. (1992). Pathway of incorporation of micro-injected lamin into the nuclear envelope. J. Cell Biol. 119, 725-735.

Goldberg, M. W. and Allen, T. D. (1992). High resolution scanning electron microscopy of the nuclear envelope; demonstration of a new, regular fibrous lattice attached to the baskets of the nucleoplasmic face of the nuclear pores. J. Cell Biol. 119, 1429-1440.

Heald, R. and McKeon, F. (1990). Mutations of phosphorylation sites in lamin A that prevent nuclear lamina disassembly in mitosis. Cell 61, 579-589.

Heitlinger, E., Peter, M., Lustig, A., Villiger, W., Nigg, E. A. and Aebi, U. (1992). The role of the head and tail domain in lamin structure and assembly: analysis of bacterially expressed chicken lamin A and truncated B2 lamins. $J$. Struct. Biol. 108, 74-91.

Hennekes, H., Peter, M., Weber, K and Nigg, E. A. (1993). Phosphorylation on protein kinase $\mathrm{C}$ sites inhibits nuclear import of lamin $\mathrm{B}_{2}$. J. Cell Biol. 120, 1293-1304.

Heslop-Harrison, J. S. and Bennett, M. D. (1990). Nuclear architecture in plants. Trends Genet. 6, 401-405.

Hochstrasser, M., Mathog, D., Gruenbaum, Y. and Sedat, J. W. (1986). Spatial organisation of chromosomes in the salivary gland nuclei of Drosophila melanogaster. J. Cell Biol. 102, 112-123.

Hoger, J. H., Krohne, G. and Franke, W. W. (1988). Amino acid sequence and molecular characterisation of murine lamin B as deduced from cDNA clones. Eur. J. Cell Biol. 47, 283-290.

Holtz, D., Tanaka, R. A., Hartwig, J. and McKeon, F. (1989). The CaaX motif of lamin A functions in conjunction with the nuclear localisation signal to target assembly to the nuclear envelope. Cell 59, 696-677.

Hozak, P., Hassen, A. B., Jackson, D. A. and Cook, P. R. (1993) Visualisation of replication factories attached to a nucleoskeleton. Cell $\mathbf{7 3}$ 361-373.

Hutchison, C. J., Cox, R. and Ford, C. C. (1988). The control of DNA synthesis in a cell-free extract that recapitulates a basic cell cycle in vitro. Development 103, 553-566.

Hutchison, C. J., Brill, D., Cox, R., Gilbert, J., Kill, I. R. and Ford, C. C. (1989). DNA replication and cell-cycle control in Xenopus egg extracts. J. Cell Sci. Suppl. 12, 197-212.

Hutchison, C. J. (1994). Local and global changes in the morphology and distribution of replication centres in rapidly expanding nuclei. Chrom. Res. (in press)

Jackson D. A. and Cook, P. R. (1988). Visualisation of a filamentous nucleoskeleton with a $23 \mathrm{~nm}$ axial repeat. EMBO J. 7, 3667-3677.

Jenkins, H. E., Holman, T., Lyon, C., Lane, E. B., Stick, R. and Hutchison, C. J. (1993). Nuclei that lack a lamina accumulate karyophilic proteins and assemble a nuclear matrix. J. Cell Sci. 106, 275-285.

Jenkins, H., Whitfield, W. G. F, Goldberg, M. W., Allen, T. D. and Hutchison, C. J. (1994). Evidence for the direct involvement of lamins in the assembly of a replication competent nucleus. Biophys. Acta Polonica (in press).

Kill, I. R., Bridger, J. M., Campbell, K. H. S., Moldonado-Codina, G. and Hutchison, C. J. (1991). The timing of the formation and usage of replicase 
cluster in S-phase nuclei of human diploid fibroblasts. J. Cell Sci. 100, 869876.

Kitten, G. T. and Nigg, E. A. (1991). The CaaX motif is required for isoprenylation, carboxyl methylation, and nuclear membrane association of lamin B2. J. Cell Biol. 113, 13-23.

Krohne, G., Waizenegger, I. and Hoger, T. H. (1989). The conserved carboxy-terminal cysteine of nuclear lamins is essential for lamin association with the nuclear envelope. J. Cell Biol. 109, 2003-2011.

Lehner, C. F., Stick, R., Eppenberger, H. M. and Nigg, E. A. (1987). Differential expression of nuclear lamin proteins during chicken development. J. Cell Biol. 105, 577-587.

Leno, G. H. and Laskey, R. A. (1991). The nuclear membrane determines the timing of DNA replication in Xenopus egg extracts. J. Cell Biol. 112, 557566.

Leno, G. H., Downes, C. S. and Laskey, R. A. (1992). The nuclear membrane prevents replication of human G2 nuclei but not G1 nuclei in Xenopus egg extract. Cell 69, 151-158.

Loewinger, L. and McKeon, F. (1988). Mutations in the nuclear lamin proteins resulting in their aberrant assembly in the cytoplasm. EMBO J. 7 2301-2309.

Lohka, M. J. and Masui, Y. (1983). Formation in vitro of sperm pronuclei and mitotic chromosomes induced by amphibian ooplasmic components. Science 220, 719-721.

Lohka M. J. and Maller, J. L. (1985). Induction of nuclear envelope breakdown, chromosome condensation and spindle formation in cell-free extracts. J. Cell Biol. 101, 518-523.

Lourim, D. and Krohne, G. (1993). Membrane-associated lamins in Xenopus egg extracts: identification of two vesicle populations. J. Cell Biol. 123, 501512.

Luderus, M. E., de Graf, A., Mattia, E., den Blaauwen, J. L., Grande, M. A., de Jong, L. and von Driel, R. (1992). Binding of matrix attachment regions to lamin $\mathrm{B}_{1}$. Cell 70, 949-959.

McKeon, F. D., Kirschner, M. W. and Caput, D. (1986). Homologies in both primary and secondary structure between nuclear envelope and intermediate filament proteins. Nature 319, 463-468.

Meier, J., Campbell, K. H. S., Ford, C. C., Stick, R. and Hutchison, C. J. (1991). The role of lamin Liii in nuclear assembly and DNA replication, in cell-free extracts of Xenopus eggs. J. Cell Sci. 98, 271-279.

Meier, J. and Georgatos, S. (1994). Type B lamins remain associated with the integral nuclear envelope protein p58 during mitosis: implications for nuclear reassembly. EMBO J. 13, 1888-1897.

Miake-Lye R. and Kirschner, M. W. (1985). Induction of early mitotic events in a cell-free system. Cell 41, 165-175.

Mills, A., Blow, J. J., White, J. G., Amos, W. B., Wilcock, D. and Laskey, R. A. (1989). Replication occurs at discrete foci spaced throughout nuclei replicating in vitro. J. Cell Sci. 94, 471-477.

Moir, R. D., Donaldson, A. E. and Stewart, M. (1991). Expression in Escherichia coli of human lamins A and C: influence of head and tail domains on assembly properties and paracrystal formation. J. Cell Sci. 99, 363-372.

Nakamura, H., Morita, T. and Sato, C. (1986). Structural organization of replicon domains during DNA synthetic phase in the mammalian nucleus. Exp. Cell Res. 165, 291-297.

Nakayasu, H. and Berezney, R. (1989). Mapping replicational sites in the eukaryotic cell nucleus. J. Cell Biol. 108, 1-11.

Newport, J. W., Wilson, K. L. and Dunphy. W. G. (1990). A laminindependent pathway for nuclear envelope assembly. J. Cell Biol. 111, 2247 2259

Newport, J. W. and Dunphy, W. G. (1992). Characterisation of the membrane binding and fusion events during nuclear envelope assembly using purified components. J. Cell Biol. 116, 295-306.

Nigg. E. A. (1989). The nuclear envelope. Curr. Opin. Cell Biol. 1, 435-440.

Peter, M., Nakagawa, J., Doree, M., Labbe, J. C. and Nigg, E. A. (1990). In vitro disassembly of the nuclear lamina and $\mathbf{M}$ phase-specific phosphorylation of lamins by cdc2 kinase. Cell 61, 591-602.

Peter, M., Heitlinger, E., Haner, M., Aebi, U. and Nigg, E. A. (1991). Disassembly of in vitro formed lamin head-to-tail polymers by cdc2 kinase. EMBO J. 10, 1535-1544.
Pfaller, R., Smythe, C. and Newport, J. W. (1991). Assembly/disassembly of the nuclear envelope membrane: cell cycle-dependent binding of nuclear membrane vesicles to chromatin in vitro. Cell 65, 209-217.

Pollard, K. M., Chan, E. K. L., Grant, B. J., Sullivan, K. F., Tan, E. M. and Glass, C. A. (1990). In vitro post-translational modification of lamin B cloned from a human T-cell line. Mol. Cell Biol. 10, 2164-2175.

Quinlan, R. A., Moir, R. D. and Stewart, M. (1989). Expression in Eschericia coli of fragments of glial fibrillary acidic protein: characterization, assembly properties and paracrystal formation. J. Cell Sci. 93, 71-83.

Rober, R.-A., Weber, K. and Osborn, M. (1989). Differential timing of nuclear lamin A/C expression in the various organs of the mouse embryo and the young animal: a developmental study. Development 105, 365-378.

Senior, A. and Gerace, L. (1988). Integral membrane proteins specific to the inner nuclear membrane and associated with the nuclear lamina. J. Cell Biol. 107, 2029-2036

Sheehan, M. A., Mills, A. D., Sleeman, A. M., Laskey, R. A. and Blow J. J. (1988). Steps in the assembly of replication-competent nuclei in a cell-free system from Xenopus eggs. J. Cell Biol. 106, 1-12.

Shelton, K. R., Cobbs, C. S., Povloshock, J. T. and Brkat, R. K. (1980) Nuclear envelope fraction proteins: isolation and comparison with the nuclear protein of the avian erythrocyte. Arch. Biochem. Biophys. 174, $177-$ 186.

Sinesky, M., Fantle, K., Trujillo, M., McLain, T., Kupfer, A. and Dalton, M. (1994). The processing pathway of prelamin A. J. Cell Sci. 107, 61-67.

Smith, D. E. and Fisher P. A. (1989). Interconversion of Drosophila nuclear lamin isoforms during the five mitotic cycles that precede gastrulation in Drosophila embryogenesis. J. Cell Biol. 108, 255-266.

Stewart, C. and Burke, B. (1987). Teratocarcinoma stem cells and early mouse embyros contain only a single major lamin polypeptide closely resembling lamin B. Cell 51, 383-392.

Stewart, M. and Whytock, S. (1988). The structure and interactions of components of nuclear envelopes from Xenopus oocyte germinal vesicles observed by heavy metal shadowing. J. Cell Sci. 90, 409-423.

Stick, R. and Hausen, P. (1985). Changes in the nuclear lamina composition during early development of Xenopus laevis. Cell 41, 191-200.

Stick, R. (1988). cDNA cloning of the developmentally regulated lamin Liii of Xenopus laevis. EMBO J. 7, 3189-3197.

Stick, R., Angres, B., Lehner, C. F. and Nigg, E. A. (1988). The fates of chicken nuclear lamin proteins during mitosis: evidence for a reversible redistribution of lamin B2 between inner nuclear membrane and elements of the endoplasmic reticulum. J. Cell Biol. 107, 397-406.

Ulitzur, N., Harel, A., Feinstein N. and Gruembaum, Y. (1992). Lamin activity is essential for nuclear envelope assembly in a Drosophila embyro cell-free system. J. Cell Biol. 119, 17-25.

Vigers, G. P. A. and Lohka, M. J. (1991). A distinct vesicle population targets membranes and pore complexes to the nuclear envelope in Xenopus eggs. J. Cell Biol. 112, 454-556.

Vigers, G. P. A. and Lohka, M. J. (1992). Regulation of nuclear envelope precursor functions during cell division. J. Cell Sci. 102, 273-284.

Vorburger, K., Lehner, C. F., Kitten, G. T., Eppenberger, H. M. and Nigg, E. A. (1989a). A second higher vertebrate B-type lamin: cDNA sequence determination and in vitro processing of chicken lamin B2. J. Mol. Biol. 208, 405-415.

Vorburger, K., Kitten, G. T. and Nigg, E. A. (1989b). Modification of nuclear lamin proteins by a mevalonic acid derivative occurs in reticulocyte lysates and requires the cysteine residue of the C-terminal CXXM motif. EMBO J. 8, 4007-4013.

Ward, G. E. and Kirschner, M. W. (1990). Identification of cell cycleregulated phosphorylation sites on nuclear lamin C. Cell 61, 561-577.

Weber, K., Plessmann, U. and Traub, P. (1989). Maturation of nuclear lamin A involves a specific carboxy-terminal trimming, which removes the polyisoprenylation site from the precursor; implications for the structure of the nuclear lamina. FEBS Lett. 257, 411-414.

Wolda, S. L. and Glomset, J. A. (1988). Evidence for modification of lamin B by a product of mevalonic acid. J. Biol. Chem. 263, 5997-6000.

Wolin, S. L., Krohne, G. and Kirschner, M. W. (1987). A new lamin in Xenopus somatic tissues displays strong homology to human lamin A. EMBO J. 6, 3809-3818. 